\title{
Compaction pressure, wall friction and surface roughness upon compaction strength of Andrographis paniculata tablets
}

\begin{abstract}
A well-known Malaysian herb, Andrographis paniculata was used in this study. This herb is famous at reducing sugar levels for diabetic patients. In this study, the herb was compressed into tablets. A compressed tablet, which is a universal form in modern medicine, needs to meet certain mechanical strength criteria in order to withstand post-compaction loading such as coating, handling, packaging and storage. The objectives of this work were to investigate the effects of compaction pressure, wall friction and surface roughness upon compaction strength of A. paniculata herb during compression. A universal testing machine with pressures ranging from 15 to $30 \mathrm{MPa}$ was used to compact the herb using a 20-mm-diameter cylindrical stainless steel uniaxial die. The tensile strength of the tablet increased as the compaction pressure increased. During compression, as the amount of powder being compressed increased, the tensile strength increased, and from the surface roughness test, the coefficient of wall friction and angle of wall friction decreased. In general, the compaction pressure, the wall friction and surface roughness plays a significant role in tabletting; hence, in producing a tough and coherent tablet.
\end{abstract}

Keyword: Wall friction, Surface roughness, Compaction pressure, Andrographis paniculata 